\title{
Protocolo Fonoaudiológico de Avaliação do Risco para Disfagia (PARD)
}

\section{Dysphagia Risk Evaluation Protocol}

\author{
Aline Rodrigues Padovani ${ }^{1}$, Danielle Pedroni Moraes ${ }^{2}$, Laura Davidson Mangili \\ Claudia Regina Furquim de Andrade ${ }^{4}$
}

\begin{abstract}
RESUMO
Objetivos: Os objetivos desse estudo foram: constituir um protocolo piloto de avaliação do risco para disfagia, visando auxiliar o fonoaudiólogo a identificar e interpretar as alterações na dinâmica da deglutição, caracterizar os sinais clínicos sugestivos de penetração laríngea ou aspiração laringo-traqueal, definir pontualmente a gravidade da disfagia e estabelecer condutas a partir dos resultados da avaliação. Métodos: O Protocolo Fonoaudiológico de Avaliação do Risco para Disfagia foi elaborado com base na literatura, segundo a identificação dos pontos comuns a todos os protocolos de avaliação da deglutição. Os pontos não comuns foram excluídos e os itens julgados relevantes foram incluídos. Resultados: O Protocolo Fonoaudiológico de Avaliação do Risco para Disfagia foi constituído por três partes: teste de deglutição da água, teste de deglutição de alimentos pastosos, classificação do grau de disfagia e condutas. Conclusão: O Protocolo Fonoaudiológico de Avaliação do Risco para Disfagia é baseado em uma proposição teórica e depende de sua aplicação populacional, em larga escala e por diferentes profissionais para que venha a se configurar como um teste validado em sua proposta. A contribuição aqui apresentada busca uma forma de contemplar, de maneira mais completa possível, a avaliação fonoaudiológica para o risco de disfagia em beira-de-leito, norteando a atuação fonoaudiológica e consolidando sua atuação baseada em evidências. A segunda fase desta pesquisa será experimental.
\end{abstract}

Descritores: Avaliação; Deglutição; Disfagia; Transtornos da deglutição; Protocolos

\section{INTRODUÇÃO}

A atuação fonoaudiológica em hospitais é relativamente recente, principalmente no que diz respeito ao acompanhamento de pacientes internados ${ }^{(1)}$, mostrando-se uma prática em expansão em vários serviços no Brasil, com aumento significativo de pesquisas nessa área ${ }^{(2)}$.

O fonoaudiólogo ingressa na equipe atuando de forma multi e interdisciplinar, com o objetivo de prevenir e reduzir complicações, a partir do gerenciamento da deglutição e da comunicação, de maneira segura e eficaz ${ }^{(3-4)}$. A contribuição

(1) Fonoaudióloga do Instituto Central do Hospital das Clínicas da Faculdade de Medicina da Universidade de São Paulo - USP - São Paulo (SP), Brasil. (2) Fonoaudióloga do Instituto Central do Hospital das Clínicas da Faculdade de Medicina da Universidade de São Paulo - USP - São Paulo (SP), Brasil.

(3) Pós-graduanda do Departamento de Fisioterapia, Fonoaudiologia e Terapia Ocupacional da Faculdade de Medicina da Universidade de São Paulo - USP - São Paulo (SP), Brasil.

(4) Livre Docente, Professora Titular do Departamento de Fisioterapia, Fonoaudiologia e Terapia Ocupacional da Faculdade de Medicina da Universidade de São Paulo - USP - São Paulo (SP), Brasil.

Trabalho realizado no Instituto Central do Hospital das Clínicas da Faculdade de Medicina da Universidade de São Paulo e no Departamento de Fisioterapia, Fonoaudiologia e Terapia Ocupacional da Faculdade de Medicina da Universidade de São Paulo - USP - São Paulo (SP), Brasil

Endereço para correspondência: Aline Rodrigues Padovani. Av. Dr. Enéas de Carvalho Aguiar, 255, $4^{\circ}$ andar, Serviço de Fisioterapia, Cerqueira César, São Paulo - SP, CEP 05403-900. E-mail: alinepadovani@ hcnet.usp.br.

Recebido em: 12/3/2007; Aceito em: 17/8/2007 da Fonoaudiologia busca ampliar as perspectivas prognósticas, com a redução do tempo de internação e a redução na taxa de re-internações por pneumonia aspirativa, contribuindo significativamente para a melhoria da qualidade de vida dos pacientes ${ }^{(5-6)}$.

Dentro deste contexto, é observada a necessidade de avaliações fonoaudiológicas pró-ativas, planejadas e controladas, estabelecendo-se métodos objetivos, princípios de avaliação consensuais e de aplicação por profissionais com expertise na área ${ }^{(7)}$.

A elaboração de protocolos que norteiem a atuação fonoaudiológica vem ao encontro destas questões, visando garantir a qualidade do que está sendo oferecido, além de permitir a aplicação do conceito de atuação baseada em evidências ${ }^{(8)}$. A fundamentação metodológica de avaliação permite que os dados sejam coletados, de maneira préestabelecida, possibilitando análise e definição de condutas. Em estudo prévio ${ }^{(5)}$, observou-se uma redução da incidência de pneumonia aspirativa em pacientes hospitalizados, a partir da aplicação de um protocolo formal de avaliação da disfagia.

A disfagia é um distúrbio da deglutição decorrente de causas neurológicas e/ou estruturais ${ }^{(4)}$. Pode ser decorrente de traumas de cabeça e pescoço, de acidente vascular encefálico, de doenças neuromusculares degenerativas, de câncer de cabeça e pescoço, de demências e encefalopatias. A disfagia mais freqüentemente reflete problemas envolven- 
do a cavidade oral, faringe, esôfago ou transição esofagogástrica. A disfagia ou dificuldade na deglutição pode resultar na entrada de alimento na via aérea, resultando em tosse, sufocação/asfixia, problemas pulmonares e aspiração. Também, gera déficits nutricionais, desidratação com resultado em perda de peso, pneumonia e morte.

O objetivo desse trabalho foi constituir a primeira fase de um protocolo piloto de avaliação do risco para disfagia, sem aplicação populacional, baseado na literatura. Tal protocolo visa auxiliar o fonoaudiólogo a identificar e interpretar as alterações na dinâmica da deglutição, caracterizar os sinais clínicos sugestivos de penetração laríngea ou aspiração laringo-traqueal, definir pontualmente a gravidade da disfagia e estabelecer condutas a partir dos resultados da avaliação.

\section{MÉTODOS}

O Protocolo Fonoaudiológico de Avaliação do Risco para Disfagia (PARD) foi elaborado com base na literatura ${ }^{(1,3,6,9-}$ ${ }^{28)}$. Levando-se em conta os protocolos já existentes, foram identificados os pontos comuns a todos, os pontos não comuns foram excluídos e itens julgados relevantes foram incluídos. Dessa forma, elaborou-se o PARD, que é constituído por três partes: teste de deglutição da água, teste de deglutição de alimentos pastosos, classificação do grau de disfagia e condutas. $\mathrm{O}$ protocolo foi submetido à avaliação de juízes (três fonoaudiólogos com experiência na área) e obteve um grau de concordância acima de $75 \%$.

\section{RESULTADOS}

Como resultado da literatura consultada, propõe-se o protocolo de risco para disfagia exposto na Figura 1.

A primeira parte do protocolo - Teste de Deglutição da Água - é composta por 11 itens. Deve ser marcada a presença ou ausência da atividade frente à quantidade de líquido oferecida. Os itens analisados são:

Escape oral anterior ${ }^{(9-10)}$ - é definido como a ocorrência de escorrimento do alimento ou líquido pelos lábios, após a captação do bolo, geralmente por insuficiência do vedamento labial. Considera-se ausência quando não há escorrimento de líquido pelas comissuras labiais, após a oferta e presença quando ocorre o escorrimento do líquido pelas comissuras labiais.

Tempo de trânsito oral ${ }^{(11-14)}$ - é definido como o tempo entre a captação completa do bolo até o início da elevação do complexo hiolaríngeo, determinada pelo disparo do reflexo de deglutição. Considera-se adequado, para o tempo máximo de quatro segundos e lento, quando o tempo de trânsito oral ultrapassa quatro segundos.

Refluxo nasal ${ }^{(10,15)}$ - é definido como escorrimento do líquido para a cavidade nasal durante a deglutição, decorrente de insuficiência no fechamento velofaríngeo. Deve ser considerada ausência, quando não há escape de líquido pelo nariz após a oferta e presença, quando ocorre escape de líquido pelo nariz após a oferta.

Número de deglutições ${ }^{(10,12,15-16)}$ - é definido como a quantidade de deglutições realizadas para completo clareamento da via digestiva após introdução do bolo na cavidade oral. A deglutição múltipla indica que ao invés deglutir o bolo em uma única massa coesa, o paciente deglute apenas uma parte deste, requerendo duas ou mais deglutições para que ocorra o completo clareamento das vias de deglutição. Deglutições múltiplas espontâneas ocorrem com freqüência em indivíduos com resíduo em cavidade oral e recessos faríngeos, podendo sinalizar dificuldade de propulsão oral, alteração de reflexo de deglutição e paresia de parede de faringe. Devese observar a presença de deglutição, por meio do monitoramento da elevação laríngea e ausculta cervical, e o número de vezes em que ela ocorre, considerando-se adequada, a presença de uma única deglutição para todas as ofertas; múltiplas, na presença de mais de uma deglutição em até um minuto após a oferta e; ausente, quando não há efetivação da deglutição, sendo necessária a interrupção do teste.

Elevação laríngea $a^{(9,11,13,15)}$ - é o termo utilizado para determinar a capacidade de excursão laríngea anterior e superior durante a deglutição, cuja dificuldade indica um aumento do risco de aspiração. A elevação laríngea adequada facilita o fechamento vertical do vestíbulo laríngeo, auxiliando na proteção de vias aéreas e na abertura da transição faringoesofágica, podendo ser monitorada com o posicionamento dos dedos indicador e médio sobre o hióide e cartilagem tireóide. O monitoramento visual e digital dessa região, também pode contribuir com interpretações sobre o desempenho oral associado ao disparo do reflexo de deglutição, inferindo o vigor da deglutição, bem como a trajetória do bolo. Considera-se adequada, a elevação laríngea que atinja, em média, dois dedos do examinador; reduzida, a elevação laríngea que atinja menos de dois dedos do examinador e; ausente, na ausência de deglutição, sendo necessária a interrupção do teste.

Ausculta cervical ${ }^{(13,17-19)}$ - é definida como a escuta dos sons associados à deglutição, por meio da utilização do estetoscópio, posicionado na região cervical. Deve ser realizada antes, durante e após a deglutição, fornecendo pistas adicionais à avaliação clínica a respeito da presença ou ausência de resíduos na faringe ou na laringe. O estetoscópio deve ser posicionado na parte lateral da junção da laringe e a traquéia, anterior à carótida. Em uma deglutição nãodisfágica, em geral, há três sons marcantes quando o bolo passa para a faringe: dois cliques audíveis acompanhados de um sopro expiratório. A ausculta dos sons da respiração (inspiração e expiração) antes da deglutição oferece um padrão consistente de comparação após a deglutição, podendo-se verificar o surgimento de ruídos. Caso o paciente apresente sensibilidade na região faríngea, também pode referir sensação de alimento parado nessa região. Outra medida avaliável é o período de apnéia da deglutição, caracterizada pela interrupção do fluxo respiratório durante a deglutição. Desta maneira, caracteriza-se ausculta cervical adequada, quando há ausência de ruídos na sequiência de expiração ou inspiração, apnéia, clunck de deglutição e expiração ou inspiração; ausculta cervical alterada antes e após a deglutição, na presença de ruídos na respiração antes da deglutição e manutenção destes ruídos de mesma frequiência após a deglutição, 


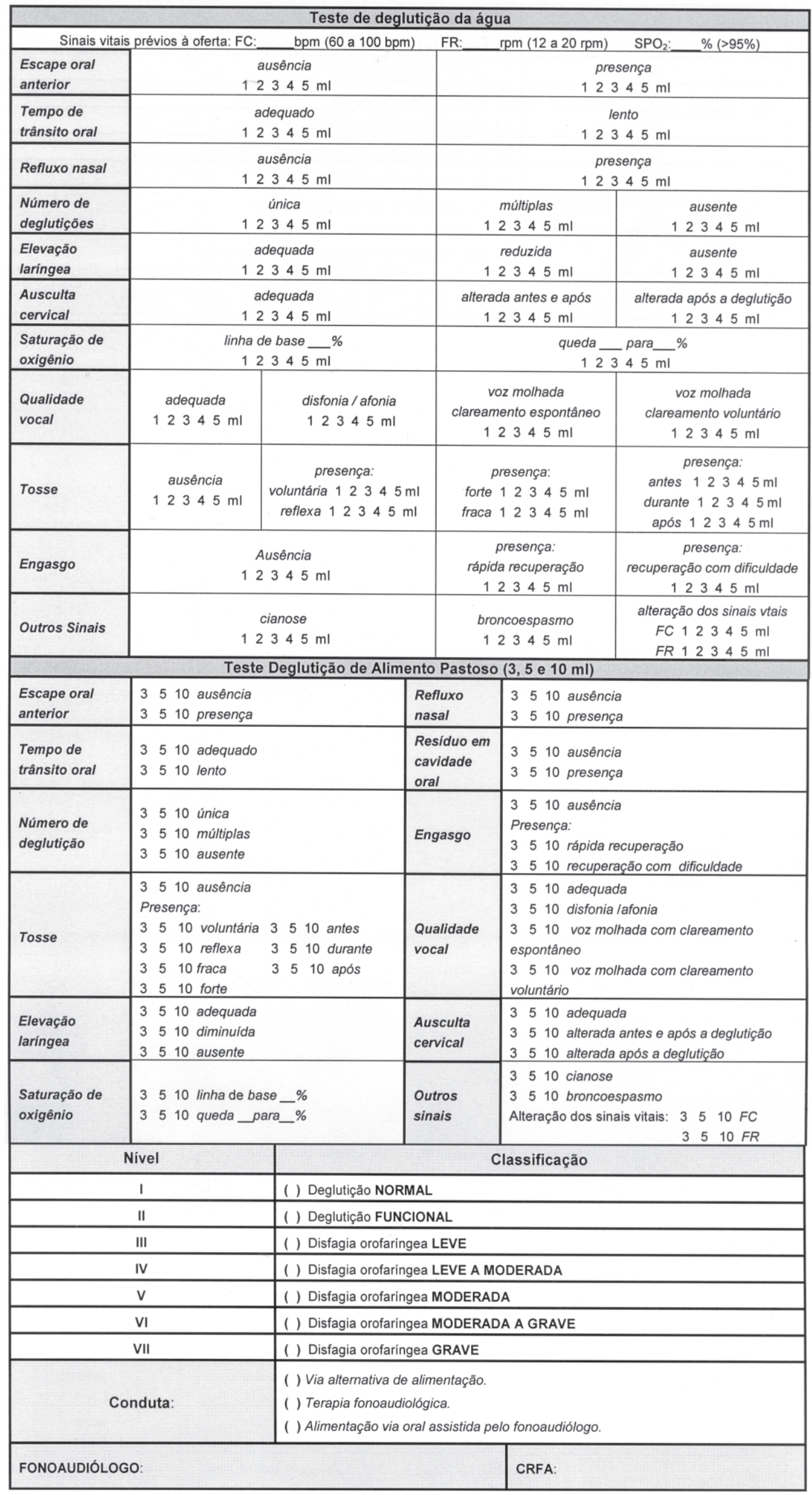

Figura 1. Protocolo Fonoaudiológico de Avaliação do Risco para Disfagia (PARD) 
e ausculta cervical alterada após a deglutição, quando ocorre presença de ruídos, não observados anteriormente, após a ausculta do clunck de deglutição.

Saturação de oxigênio $\left(\mathrm{SpO} \mathrm{O}^{(15,20-21)}\right.$ - é definida como a porcentagem de oxigênio arterial na corrente sanguínea, por meio da mensuração da oximetria de pulso. O uso do oxímetro de pulso para detectar aspiração baseia-se na hipótese que a aspiração do alimento causaria um reflexo de broncoespasmo, diminuindo a perfusão-ventilatória e provocando a queda na saturação de oxigênio. Considera-se adequada para manutenção ou redução de até $4 \%$ da linha de base do paciente e queda de saturação para redução maior que $4 \%$ da linha de base após a oferta.

Qualidade vocal ${ }^{(6,9,11,13,15,22-23)}$ - é o termo empregado para designar o conjunto de características que identificam uma voz e visa identificar a presença ou ausência de voz molhada após oferta de alimento ou líquido, por meio da comparação pré e pós-deglutição. A voz molhada é um termo que descreve o som borbulhante produzido na fonação de um " $e$ " prolongado, indicativo de estase de secreções, líquidos ou alimentos no vestíbulo laríngeo, podendo detectar a penetração silente nas pregas vocais. Na presença de voz molhada, observa-se a percepção do indivíduo por meio da resposta de tosse ou pigarro espontâneos, indicando sensibilidade laríngea adequada. O pigarro é produzido por aproximação das pregas vocais e pode ser percebido como um "ahem". Pacientes que não percebem a sua qualidade vocal molhada apresentam sinais de diminuição da sensibilidade laríngea e podem ter mais chances de aspiração. Portanto, considera-se adequada, a ausência de rouquidão, soprosidade e voz molhada; rouquidão elou afonia, na presença destas características e; voz molhada na presença de um som borbulhante na voz após a oferta da consistência. É necessário dar relevância à voz molhada com clareamento laríngeo espontâneo ou qualidade vocal rouco-soprosa, em associação com outras alterações observadas durante a avaliação, pois estas características estão freqüentemente associadas ao aumento do risco de aspiração.

Tosse $e^{(3,6,9-11,13,15,17,23-24)}-\mathrm{A}$ tosse é uma resposta reflexa do tronco cerebral que protege a via aérea contra a entrada de corpos estranhos, podendo também ser produzida voluntariamente. A tosse reflexa durante ou após a deglutição é um clássico sinal de aspiração por disfagia orofaríngea, sendo indicador da existência de sensibilidade na região laríngea e da habilidade de expectoração, embora sua presença não seja sinônimo de clareamento de via aérea. A tosse voluntária refere-se à tosse produzida sob comando e não está relacionada à aspiração. Avalia-se para determinar a habilidade do paciente de expelir material da via aérea durante a oferta de líquido ou pastoso, caso necessário. Na presença de tosse, são consideradas três características principais: o desencadeamento (reflexo ou voluntário), a força (forte ou fraca) e o momento em que ocorre (antes, durante ou depois). Assim, considera-se ausência, quando não ocorre tosse reflexa ou voluntária durante a avaliação; tosse reflexa, na presença de tosse sem solicitação; tosse voluntária, na presença de tosse sob solicitação, geralmente necessária após observação de ausculta cervical alterada ou voz molhada sem clareamento espontâneo; tosse forte ou eficaz, na presença de tosse capaz de mobilizar estase de secreção e clarear a via aérea; tosse fraca ou ineficaz, na presença de tosse incapaz de mobilizar estase de secreção em via aérea; tosse antes $d a$ deglutição, na presença de tosse após a captação do bolo e antes do disparo do reflexo de deglutição; tosse durante a deglutição, na presença de tosse imediatamente após a ocorrência do reflexo de deglutição e; tosse após a deglutição, na presença de tosse em até um minuto após o disparo do reflexo de deglutição.

Engasgo ${ }^{(9,11,17,23,25-26,28)}$ - definido como obstrução do fluxo aéreo, parcial ou completo, decorrente da entrada de um corpo estranho nas vias aéreas inferiores, podendo levar à cianose e asfixia. Considera-se ausência, quando não ocorre engasgo; presença com rápida recuperação, na ocorrência de tosses durante a deglutição, sem episódio de cianose e com rápida recuperação da frequiência respiratória de base e; presença com difícil recuperação, na ocorrência de tosses durante a deglutição, podendo ocorrer cianose, com difícil recuperação da frequiência respiratória de base.

\section{Outros sinais}

- Cianose $e^{(26)}$ - é definida como a coloração azulada da pele, causada por pressões excessivas de hemoglobina desoxigenada nos plexos capilares e venosos. A presença de cianose constitui um dos sinais clínicos mais comuns dos diferentes graus de insuficiência respiratória. Considera-se cianose, na presença deste sinal.

- Broncoespasmo ${ }^{(15,20-21,26)}$ - é definido pela dificuldade respiratória causada por uma constrição repentina dos músculos das paredes brônquicas. Alguns autores hipotetizam que a aspiração do alimento pode causar reflexos de broncoespasmo, ocasionando, portanto, um estreitamento de vias aéreas inferiores e um aumento da produção do muco, que leva à insuficiência respiratória, tosse e hipoxia, percebido por meio de sibilos na respiração. Considerase broncoespasmo, na presença deste sinal, observado por meio da escuta de sibilos inspiratórios e/ou expiratórios após a oferta.

- Alteração dos sinais vitais

a) Freqüência cardíaca $(\mathrm{FC})^{(1,19,26)}$ - é a medida da quantidade de batimentos cardíacos por minutos. Permite verificar se ocorrem mudanças bruscas na freqüência dos batimentos cardíacos durante a função de deglutição. Considera-se alteração na freqüência cardíaca, na ocorrência de queda ou aumento excessivo da freqüência cardíaca, tendo como base a faixa de normalidade de 60 a 100 batimentos por minuto (bpm).

b) Frequiência respiratória $(\mathrm{FR})^{(1,13,17,19,26)}$ - é a medida da quantidade de ciclos respiratórios (inspiração e expiração) por minuto. A incoordenação entre a deglutição e a respiração aumenta o risco de aspiração em pacientes taquipneicos ou dispneicos, pois estes podem não ser capazes de tolerar períodos maiores ou mesmo curtos de apnéia durante a deglutição. Espera-se que pacientes idosos taquipneicos sejam incapazes de fazer o ajuste da deglutição na expiração durante a deglutição de líquidos ou saliva, tendo uma alta freqüência de aspiração quando 
comparados com idosos saudáveis ou adultos jovens ${ }^{(13)}$. Considera-se alteração na freqüência respiratória, na ocorrência de queda ou aumento excessivo da frequiência respiratória, tendo como base a faixa de normalidade de 12 a 20 respirações por minuto (rpm).

A segunda parte do protocolo - Teste de Deglutição de Alimento Pastoso - é composta por 12 itens. Os 11 itens analisados no teste da água são reavaliados, tornando-se necessário avaliar também a ocorrência de resíduo de alimento em cavidade oral. Nessa parte do PARD, também são ajustadas as quantidades de $\mathrm{ml}$ oferecidos, pela variação da consistência do alimento.

Resíduo em cavidade oral após deglutição ${ }^{(10,17)}$ - é o acúmulo de alimento em vestíbulo anterior, lateral, assoalho bucal e/ou superfície lingual após a deglutição. Adota-se a inspeção da cavidade oral, considerando normais resíduos de até aproximadamente $25 \%$ do bolo ofertado. Considerase ausência, quando não se observa presença de resíduos do alimento em cavidade oral após a deglutição e presença, quando se observa presença de resíduos do alimento em cavidade oral após a deglutição.

Tempo de trânsito oral ${ }^{(11-14)}$ - para o teste de deglutição com alimento pastoso considera-se adequado, para o tempo máximo de 17,5 segundos e lento, quando o tempo de trânsito oral ultrapassa 17,5 segundos.

A terceira parte do protocolo - Classificação do Grau de Disfagia e Condutas - é composta por cinco níveis de classificação da disfagia e três tipos de condutas, baseados na Escala de Gravidade e Resultados da Disfagia ${ }^{(27)}$ e na Escala de Gravidade da Disfagia ${ }^{(28)}$. A classificação é proposta conforme a gravidade do distúrbio de deglutição e direciona o fonoaudiólogo na tomada de condutas. Assim, deve-se seguir o raciocínio clínico proposto nas especificações dos itens do protocolo, de acordo com os sinais apresentados pelo paciente. Para a classificação da disfagia, é necessário que o paciente apresente pelo menos um sinal que o diferencie do nível anterior.

Nível I. Deglutição normal - Normal para ambas as consistências e em todos os itens avaliados. Nenhuma estratégia ou tempo extra é necessário. A alimentação via oral completa é recomendada.

Nível II. Deglutição funcional - Pode estar anormal ou alterada, mas não resulta em aspiração ou redução da eficiência da deglutição, sendo possível manter adequada nutrição e hidratação por via oral ${ }^{(3)}$. Assim, são esperadas compensações espontâneas de dificuldades leves, em pelo menos uma consistência, com ausência de sinais de risco de aspiração. A alimentação via oral completa é recomendada, mas pode ser necessário despender tempo adicional para esta tarefa.

Nível III. Disfagia orofaríngea leve - Distúrbio de deglutição presente, com necessidade de orientações específicas dadas pelo fonoaudiólogo durante a deglutição. Necessidade de pequenas modificações na dieta; tosse e/ou pigarro espontâneos e eficazes; leves alterações orais com compensações adequadas.

Nivel IV. Disfagia orofaríngea leve a moderada - Existência de risco de aspiração, porém reduzido com o uso de manobras e técnicas terapêuticas. Necessidade de supervisão esporádica para realização de precauções terapêuticas; sinais de aspiração e restrição de uma consistência; tosse reflexa fraca e voluntária forte. O tempo para a alimentação é significativamente aumentado e a suplementação nutricional é indicada.

Nivel V. Disfagia orofaríngea moderada - Existência de risco significativo de aspiração. Alimentação oral suplementada por via alternativa, sinais de aspiração para duas consistências. $\mathrm{O}$ paciente pode se alimentar de algumas consistências, utilizando técnicas específicas para minimizar o potencial de aspiração e/ou facilitar a deglutição, com necessidade de supervisão. Tosse reflexa fraca ou ausente.

Nível VI. Disfagia orofaríngea moderada a grave - Tolerância de apenas uma consistência, com máxima assistência para utilização de estratégias, sinais de aspiração com necessidade de múltiplas solicitações de clareamento, aspiração de duas ou mais consistências, ausência de tosse reflexa, tosse voluntária fraca e ineficaz. Se o estado pulmonar do paciente estiver comprometido, é necessário suspender a alimentação por via oral.

Nível VII. Disfagia orofaríngea grave - Impossibilidade de alimentação via oral. Engasgo com dificuldade de recuperação; presença de cianose ou broncoespasmos; aspiração silente para duas ou mais consistências; tosse voluntária ineficaz; inabilidade de iniciar deglutição.

As condutas devem ser dadas de acordo com a classificação da disfagia e incluem a indicação de: (a) via alternativa de alimentação, como as sondas enterais e gástricas; (b) terapia fonoaudiológica, podendo ser direta (com alimento) e/ ou indireta (sem alimento) e; (c) alimentação via oral assistida pelo fonoaudiólogo, de acordo com a seleção das consistências. As propostas de conduta baseadas na classificação da disfagia ${ }^{(27-28)}$ estão a seguir:

- Para os níveis I e II, a conduta será (c);

- Para os níveis III, IV e V, a conduta será (a) + (b) + (c) e

- Para os níveis VI e VII, a conduta será (a) + (b).

\section{Aplicação do protocolo}

Para a aplicação do protocolo de risco da disfagia, são necessários: estetoscópio, equipamento de oximetria de pulso, equipamentos de monitorização de sinais vitais, $15 \mathrm{ml}$ de água potável, $54 \mathrm{ml}$ de alimento pastoso fino, seringas de 5 e $10 \mathrm{ml}$ e uma colher de sobremesa.

Antes de iniciar a aplicação do protocolo de risco, é necessário que o fonoaudiólogo esteja atento às condições clínicas do paciente. É imprescindível que este esteja alerta, acordado e capaz de receber líquidos e alimentos na boca ${ }^{(6,13)}$. Pacientes extremamente letárgicos ou que apresentam níveis inconsistentes de alerta são de alto risco para aspiração ${ }^{(29)}$.

Há de se compreender também o quadro clínico motor geral, de linguagem e de fala do indivíduo, já que estas manifestações podem interferir no programa de reabilitação, na definição das técnicas terapêuticas a serem selecionadas, bem como na independência alimentar do indivíduo ${ }^{(15)}$. Além disso, possuem importante correlação com os diferentes graus de comprometimento da disfagia orofaríngea e podem con- 
tribuir para definir o prognóstico e facilitar a interpretação dos achados da avaliação.

$\mathrm{O}$ protocolo tem início com o teste da água, realizado por meio da oferta gradativa de 1 a $5 \mathrm{ml}$ de água na seringa. Solicita-se que o paciente sugue a água da seringa enquanto o avaliador empurra o êmbolo suavemente. Então, o paciente é orientado a deglutir. Desta maneira, o indivíduo prepara a cavidade oral para o recebimento do líquido, prevenindose contra escape prematuro para a faringe ${ }^{(17)}$.

Em seguida, é realizado o teste de deglutição do alimento pastoso, por meio do fracionamento do alimento por meio da seringa de $10 \mathrm{ml}$, gradativamente, em 3,5 e $10 \mathrm{ml}$. O alimento fracionado é colocado na colher de sobremesa e o paciente é orientado a capturar o alimento da colher e degluti-lo. O procedimento é repetido três vezes para cada gradação, observando-se a uniformidade do desempenho.

\section{DISCUSSÃO}

De acordo com a literatura ${ }^{(10)}$, uma boa avaliação da disfagia deve incluir testes que diagnostiquem alterações nas funções orais, como exemplo, aumento no trânsito oral ou deglutição incompleta do bolo, alterações na qualidade vocal, disartria, alteração no reflexo de gag, tosse voluntária ineficiente, redução da elevação laríngea durante a deglutição de saliva, alteração de sensibilidade na laringe e alterações no teste de água. Esses testes devem ser breves, não invasivos, apresentar baixo risco para o paciente e identificar os sintomas da disfagia ${ }^{(24)}$. A avaliação fonoaudiológica em beirade-leito não pode ser muito extensa, mas deve ser capaz de identificar os pacientes potencialmente de risco para disfagia, para que se possa atuar de forma pró-ativa à possíveis complicações hospitalares.

$\mathrm{O}$ teste da água tem sido descrito com freqüência em pesquisas da área ${ }^{(9,11,17,23,25,29)}$. Um dos pontos positivos do teste é o fato de que a aspiração de pequena quantidade de água é provavelmente segura, sendo relatada ausência de pneumonia em testes com animais ${ }^{(17)}$. Artigos recentes demonstraram $70 \%$ de sensibilidade e $88 \%$ de especificidade para o teste da água ${ }^{(17)}$ e $100 \%$ de sensibilidade e $71 \%$ de especificidade para o teste de água em conjunto com a monitoração da oximetria de pulso $^{(30)}$.
Em relação à consistência do alimento, encontramos que as consistências líquida e pastosa são processadas e deglutidas de maneiras diferentes ${ }^{(17)}$. Assim, uma avaliação padronizada deve incorporar testes de deglutição com ambas as consistências. Há evidências de $72 \%$ de sensibilidade e $62 \%$ de especificidade para o teste com alimento pastoso, sendo que, em associação com o teste da água, a sensibilidade aumentou para $90 \%{ }^{(17)}$. A literatura também ressalta que os volumes de 5 e $10 \mathrm{ml}$ na avaliação do alimento pastoso são mais funcionais para a identificação dos sinais sugestivos de penetração laríngea e aspiração, bem como para facilitar a interpretação e definição de $\operatorname{conduta}^{(15)}$.

A literatura aponta que os fonoaudiólogos são os profissionais mais habilitados para avaliar e tratar as alterações de deglutição ${ }^{(3-4)}$. Para exercer plenamente essa função, o fonoaudiólogo deve estar atento e preocupado em agir de forma responsável e comprovada. Basear-se em protocolos específicos permeia esses aspectos e permite atuar de forma planejada, documentando seus procedimentos, sedimentando e avaliando suas práticas e padrões de trabalho.

\section{CONCLUSÃO}

O PARD é baseado numa proposição teórica e depende de sua aplicação populacional, em larga escala e por diferentes profissionais para que venha a se configurar como um teste validado em sua proposta. A contribuição aqui apresentada busca uma forma de contemplar de maneira mais completa possível a avaliação fonoaudiológica para risco de disfagia em beira-de-leito, norteando a atuação fonoaudiológica e consolidando sua atuação baseada em evidências. Cabe à segunda fase deste trabalho a pesquisa experimental, com o controle dos sujeitos e análise estatística dos dados.

\section{AGRADECIMENTOS}

Agradecemos à Prof ${ }^{a}$. Dra. Letícia Lessa Mansur, à mestranda Fga. Priscila Esteves Ciocchi, à Fga. Fernanda Pereira de Camargo e à doutoranda Fga. Mara de Oliveira Rodrigues Luiz Dantas pela importante colaboração na elaboração do protocolo.

\begin{abstract}
Purpose: The aim of this study was to constitute a pilot protocol proposal to risk identification of dysphagia. This protocol is proposed to identify and to interpret the swallowing disorders, to characterize the penetration or aspiration clinical signals, to define promptly the dysphagia severity and to establish the management based in the results of the evaluation. Methods: The Dysphagia Risk Evaluation Protocol is based in extensive literature about dysphagia. The common points were maintained and the points with no literature consensus were dismissed. Results: The Dysphagia Risk Evaluation Protocol is constituted by three parts: water swallowing test, puree deglutition test, dysphagia severity scale and management. Conclusion: The Dysphagia Risk Evaluation Protocol is based in a theoretical proposition and depends of its application on population basis for its validation. Also contributes to a full beside evaluation that guides the speech-language pathologists and consolidate the evidence-based practice. The next step of this research will be experimental.
\end{abstract}

Keywords: Evaluation; Deglutition; Dysphagia; Deglutition disorders; Protocols 


\section{REFERÊNCIAS}

1. Barros APB, Martins NMS, Carrara-de Angelis E, Fúria CLB, Lotfi CJ. Atuação fonoaudiológica em unidade de terapia intensiva. In: Fundação Oncocentro de São Paulo. Comitê de Fonoaudiologia em Cancerologia. Fonoaudiologia em cancerologia. São Paulo: FOSP; 2000.

2. Furia CLB. Disfagias mecânicas. In: Ferreira LP, Befi-Lopes DM, Limongi SCO, organizadores. Tratado de fonoaudiologia. São Paulo: Editora Roca; 2004. p. 386-404

3. American Speech-Language-Hearing Association. Roles of speechlanguage pathologists in swallowing and feeding disorders: technical report. ASHA Desk Reference. 2002;3:181-99.

4. American Speech-Language-Hearing Association. Model Medical Review Guidelines for Dysphagia Services [monograph on the Internet] 2004 [Revision to DynCorp 2001 FTRP by ASHA]. [cited 2007 Mar 3]. Available from: URL: http://www.asha.org/NR/rdonlyres/5771B0F7D7C0-4D47-832A-86FC6FEC2AE0/0/DynCorpDysph HCEC.pdf

5. Hinchey JA, Shepard T, Furie K, Smith D, Wang D, Tonn S; Stroke Practice Improvement Network Investigators. Formal dysphagia screening protocols prevent pneumonia. Stroke. 2005;36(9):1972-6.

6. Smith Hammond CA, Goldstein LB. Cough and aspiration of food and liquids due to oral-pharyngeal dysphagia: ACCP evidence-based clinical practice guidelines. Chest. 2006;129(1 Suppl):154S-168S.

7. Andrade CRF. Pesquisa em gagueira: considerações fonoaudiológicas. In: XIII Congresso Brasileiro de Fonoaudiologia, 2005, Rev Soc Bras Fonoaudiol [CD-ROM]. 2005: Supl. especial. [Apresentado no XIII Congresso Brasileiro de Fonoaudiologia, 2005].

8. Apel K, Self T. Evidence-based practice: the marriage of research and clinical services. The Asha Leader Online [serial on the Internet]. 2003 Sept 9 [about 4 p.]. [cited 2007 Mar 3]. Available from: URL: http:// www.asha.org/about/publications/leader-online/archives/2003/q3/ 030909.htm.

9. Nishiwaki K, Tsuji T, Liu M, Hase K, Tanaka N, Fujiwara T. Identification of a simple screening tool for dysphagia in patients with stroke using factor analysis of multiple dysphagia variables. J Rehabil Med. 2005;37(4):247-51.

10. Logemann JA. Evaluation and treatment of swallowing disorders. 2nd ed. Texas: Pro-Ed; 1998.

11. Leslie P, Carding PN, Wilson JA. Investigation and management of chronic dysphagia. BMJ. 2003;326(7386):433-6. Comments in: BMJ. 2003;326(7399):1147-8. BMJ. 2003;326(7399):1148.

12. Marchesan IQ. O que se considera normal na deglutição. In: Jacobi JS, Levy DS, Silva LMC. Disfagia: avaliação e tratamento. Rio de Janeiro: Revinter; c2003.

13. Goldsmith T. Evaluation and treatment of swallowing disorders following endotracheal intubation and tracheostomy. Int Anesthesiol Clin. 2000;38(3):219-42.

14. Santoro PP, Tsuji DH, Lorenzi MC, Ricci F. A utilização da videoendoscopia da deglutição para a avaliação quantitativa da duração das fases oral e faríngea da deglutição na população geriátrica. Arq Int Otorrinolaringol. 2003;7(3):181-7.
15. Silva RG. Disfagia orofaríngea pós-acidente vascular encefálico. In: Ferreira LP, Befi-Lopes DM, Limongi SCO, organizadores. Tratado de fonoaudiologia. São Paulo: Roca; 2004. p.354-369.

16. Ertekin C, Aydogdu I, Yüceyar N. Piecemeal deglutition and dysphagia limit in normal subjects and in patients with swallowing disorders. J Neurol Neurosurg Psychiatry. 1996;61(5):491-6.

17. Tohara H, Saitoh E, Mays KA, Kuhlemeier K, Palmer JB. Three tests for predicting aspiration without videofluorography. Dysphagia. 2003;18(2):126-34.

18. McKaig TN. Ausculta cervical e torácica. In: Furquim AM, Santini CS, organizadores. Disfagias orofaríngeas. Carapicuíba: Pro-Fono; 1999. p. 171-87.

19. Luiz MOR. Rotinas em pacientes traqueostomizados: avaliação fonoaudiológica. In: Auler Jr JOC, Oliveira AS, organizadores. Pósoperatório de cirurgia torácica e cardiovascular: rotinas do Incor Instituto do Coração/HCFMUSP. Porto Alegre: Artmed; 2004.

20. Collins MJ, Bakheit AM. Does pulse oximetry reliably detect aspiration in dysphagic stroke patients? Stroke. 1997;28(9):1773-5.

21. Ramsey DJ, Smithard DG, Kalra L. Early assessments of dysphagia and aspiration risk in acute stroke patients. Stroke. 2003;34(5):1252-7.

22. Ryu JS, Park SR, Choi KH. Prediction of laryngeal aspiration using voice analysis. Am J Phys Med Rehabil. 2004;83(10):753-7.

23. DePippo KL, Holas MA, Reding MJ. Validation of the 3-oz water swallow test for aspiration following stroke. Arch Neurol. 1992;49(12):1259-61. Comment in: Arch Neurol. 1994;51(2):119-20.

24. Logemann JA, Veis S, Colangelo L. A screening procedure for oropharyngeal dysphagia. Dysphagia. 1999;14(1):44-51.

25. Wu MC, Chang YC, Wang TG, Lin LC. Evaluating swallowing dysfunction using a 100-ml water swallowing test. Dysphagia. 2004;19(1):43-7.

26. Guyton AC. Fisiologia humana e mecanismos das doenças. 3a ed. Rio de Janeiro: Guanabara Koogan; 1986.

27. O'Neil KH, Purdy M, Falk J, Gallo L. The dysphagia outcome and severity scale. Dysphagia. 1999;14(3):139-45.

28. Gramigna GD. How to perform video-fluoroscopic swallowing studies. GI Motility Online. [serial on the Internet]. 2006; [about 9p.] [cited 2007 Mar 3] Available from: URL: http://www.nature.com/gimo/contents/pt1/ full/gimo95.html\#t6.

29. Vergis EN, Brennen C, Wagener M, Muder RR. Pneumonia in long-term care: a prospective case-control study of risk factors and impact on survival. Arch Intern Med. 2001;161(19):2378-81.

30. Lim SH, Lieu PK, Phua SY, Seshadri R, Venketasubramanian N, Lee $\mathrm{SH}$, Choo PW. Accuracy of bedside clinical methods compared with fiberoptic endoscopic examination of swallowing (FEES) in determining the risk of aspiration in acute stroke patients. Dysphagia. 2001;16(1):16. 